\title{
EchoGéo
}

49 | 2019

Les corridors de développement

\section{Sur la route de La mondialisation des pauvres}

Entretien avec Armelle Choplin et Olivier Pliez, par Jean-Fabien Steck

Armelle Choplin, Olivier Pliez et Jean-Fabien Steck

\section{(2) OpenEdition}

Journals

Édition électronique

URL : https://journals.openedition.org/echogeo/18161

DOI : 10.4000/echogeo.18161

ISSN : 1963-1197

Éditeur

Pôle de recherche pour l'organisation et la diffusion de l'information géographique (CNRS UMR 8586)

Référence électronique

Armelle Choplin, Olivier Pliez et Jean-Fabien Steck, «Sur la route de La mondialisation des pauvres », EchoGéo [En ligne], 49 | 2019, mis en ligne le 25 octobre 2019, consulté le 11 août 2021. URL : http:// journals.openedition.org/echogeo/18161; DOI : https://doi.org/10.4000/echogeo.18161

Ce document a été généré automatiquement le 11 août 2021.

EchoGéo est mis à disposition selon les termes de la licence Creative Commons Attribution - Pas d'Utilisation Commerciale - Pas de Modification 4.0 International (CC BY-NC-ND) 


\title{
Sur la route de La mondialisation des
}

\section{pauvres}

\author{
Entretien avec Armelle Choplin et Olivier Pliez, par Jean-Fabien Steck \\ Armelle Choplin, Olivier Pliez et Jean-Fabien Steck
}

\section{RÉFÉRENCE}

Choplin A., Pliez O., 2018. La mondialisation des pauvres : loin de Wall Street et de Davos.

Paris, Seuil, $128 \mathrm{p}$.

1 Alors que ce numéro d'EchoGéo est consacré à l'étude des corridors de développement, Sur l'écrit propose à la fois un contrepoint et un complément en proposant un échange avec les auteurs de La Mondialisation des pauvres, paru l'an dernier.

À propos de la genèse du livre

Jean-Fabien Steck (JFS) : Si depuis plusieurs années cette thématique est présente dans vos travaux de recherche, comment s'est-elle peu à peu imposée à partir de vos travaux antérieurs, portant à la fois sur les réseaux urbains, sur les dynamiques des villes et sur les mouvements migratoires?

Armelle Choplin et Olivier Pliez (AC\&OP) : L'idée était d'abord de travailler dans les années 2000 autour des circulations entre l'Afrique subsaharienne, l'Europe et la Méditerranée. En travaillant sur ces questions, on s'est rendu compte que la part des migrants qui venait en Europe était très faible par rapport à toutes ces autres personnes qui circulaient dans ces régions et qui n'allaient pas vers l'Europe, et qui parfois avaient des connexions avec d'autres espaces comme l'Asie. C'est ainsi que l'on a commencé à s'intéresser non pas aux migrations à destination de l'Europe mais à ces circulations qui connectaient des espaces parfois inattendus avec des routes qui étaient bien moins étudiées.

C'est un travail que l'on a conduit à l'époque notamment avec Sylvie Bredeloup, Jérôme Lombard, Sophie Bava en réponse à la surmédiatisation des migrations à destination de l'Europe qui occultait ces autres circulations. Il y a eu un basculement 
vers la moitié des années 2000 et nous nous sommes penchés sur ces autres circulations, qui s'inscrivaient sur le temps long. Nous analysions plutôt les circulations de ces individus que les migrations, et la façon dont ils transformaient les lieux par lesquels ils circulaient. On a pu observer des places marchandes, avec leurs commerçants, fonctionnelles même après la fin du flux migratoire. Ils sont restés des lieux de circulation à une plus vaste échelle. Les espaces sahariens et sahéliens proposent de bons exemples : si certaines routes se sont fermées, comme celles via le Niger ou la Libye, des places marchandes sont elles restées importantes.

À propos du terrain

JFS. Dans le livre, l'enquête de terrain apparait comme la source majeure de vos analyses et de vos réflexions. Certains terrains parmi ceux que vous avez pratiqués posent de réelles questions méthodologiques : comment enquête-t-on sur la route, sur la circulation, sur le mouvement?

AC \& OP. On a emprunté certaines routes, avec les mêmes moyens de transport que les circulants, notamment les bus, ce qui permettait d'échanger avec ces personnes durant ces temps d'attente, ces temps morts que sont le trajet, les attentes dans les gares routières, au moment des passages de frontières, et finalement c'est certainement dans ces temps-morts là que les gens sont les plus disposés à parler, plutôt que quand ils sont occupés par des transactions dans des places marchandes. Nous avons donc suivi ces routes, parfois même avec des marchands que nous accompagnions. En outre, les individus rencontrés partageaient leurs contacts dans les places marchandes, à qui nous allions ensuite rendre visite et qui nous permettaient de faire le lien entre ces routes et ces lieux.

Les terrains sont aussi des lieux et des routes que l'on a revisités : souvent nous sommes retournés dans ces espaces que nous avions observé une première fois au début des années deux mille, puis vers les années 2008-2010 et enfin récemment, pour ceux qui restent accessibles. Ce sont donc des espaces que l'on a vu évoluer, changer. Que l'on a reconnu ensemble, et avec d'autres collègues de l'équipe, en Egypte, au Soudan, en Algérie, en Mauritanie, au Mali, Sénégal, Burkina, Côte d'Ivoire, et plus récemment au Bénin et au Togo. Nous avons travaillé avec la même méthodologie que nous pouvions ensuite re-déployer individuellement. Il y a donc des terrains personnels et des terrains que l'on a visité et revisité ensemble.

À propos des lieux

JFS. Dès les premières pages, la notion de «lieu » apparait centrale. Comment l'articulezvous à l'idée de la réduction de la distance, au principe même de cette dernière et d'une certaine façon de la mondialisation. Permet-elle de dépasser la notion d'enclavement?

AC \& OP. Le lieu est entendu comme une unité spatiale élémentaire. Il est aussi chargé de sens par ceux qui le fréquente. Michel Lussault, quelques mois auparavant parlait des hyper-lieux. Nous parlons de lieux plus banals mais très connectés. On questionne d'abord les lieux pour apprécier ce qui y relève du mouvement. Des positions frontalières, des toponymes, des fonctions urbaines, croisées avec des données éparses donnent une première impression. Celle-ci est alors renseignée par des échanges avec des chalands, des commerçants, des contrebandiers, des affréteurs... Toutes personnes qui permettent de reconstituer les bribes d'une chaîne d'échange. En portant attention aux autres lieux avec lesquels ils sont connectés, on peut progressivement se faire une idée de l'étendue de la chaîne et identifier l'échelle et les niveaux à laquelle elle renvoie. Le lieu est dans cette optique une unité spatiale 
élémentaire reliée à d'autres lieux par une route. Il y a là une dimension matérielle : ce qui est bâti (entrepôts, marchés) dont on peut mesurer l'emprise et ainsi saisir, même selon une approche qualitative, le « poids » de ce lieu dans la chaîne d'échange et les infrastructures d'échanges. Il y a aussi une dimension que l'on rapprocherait du lieu anthropologique qui, saisie par des observations et des entretiens, donne une image de la «densité relationnelle» (l'expression est de Alain Tarrius) traduite localement.

Ce sont des lieux qui sont considérés comme enclavés, périphériques, en marge, mais selon le point de vue que l'on adopte on peut basculer et considérer que ce sont des lieux très connectés, voire ultra-connectés pour certains. L'enclavement est vraiment une notion relative. Et notre idée, dans ce livre, c'était vraiment l'idée d'aller voir la mondialisation au plus près des pauvres et aller voir de quelle manière ces espaces que l'on croit périphériques sont pleinement ancrés dans les dynamiques de la mondialisation. Il en va ainsi de El Eulma, cet espace de souk qui est à l'intersection de deux routes algériennes et on se rend compte qu'il y a des gens de toute l'Algérie mais aussi de Tunisie qui viennent s'y approvisionner. Si on prend les marchés de gros, notamment entre les grandes villes ouest-africaines, on est vraiment dans des périphéries et des interstices urbains, et pourtant ce ne sont pas du tout des lieux enclavés, bien au contraire. Même s'ils ne sont pas forcément accessibles, ils drainent des gens qui ont parcouru des distances incroyables, que l'on calcule d'ailleurs en temps et pas vraiment en kilomètres.

En parlant de transport, on observe une redéfinition des trajectoires: moins à destination de l'Europe, mais plus à destination du monde arabe et de l'Asie, notamment via les hubs du Golfe ou, pour l'Afrique de l'Ouest en prenant appui sur le hub de la Royal Air Maroc à Casablanca. La baisse de ces coûts joue beaucoup sur ces nouveaux échanges, en plus les questions géopolitiques et de la fermeture de l'Europe qu'il convient, bien évidemment, de ne pas minorer. A l'échelle régionale, le prix des douanes joue également un rôle important sur la fréquentation et le dynamisme de certaines routes : ainsi sur le Golfe de Guinée, le montant des tarifs douaniers est inférieur à Lomé qu'il ne l'est à Cotonou ou à Lagos. Il en résulte une concentration de certains flux vers la capitale togolaise, permise également par les conditions de circulation sur le corridor côtier.

JFS. Le chapitre 2 explore quelques-uns de ces lieux. Comment ont-ils été choisis ? Comment ont-ils été mis en relation? Quels sens donnez-vous à une géographie de filière?

AC \& OP. Nous essayons d'insister sur le fait que la mise en relation prend du temps. Elle relève de dynamiques globales: basculements géopolitiques, crises ou croissances économiques, mais aussi entrepreneurs qui se construisent dans ce cadre transnational de plus en plus étendu et complexe. La Chine a, à un moment, été une destination allant de soi, simplement parce que les commerçants y allaient. Sur place apparaissait des comptoirs co-construits par leur fréquentation et l'installation de quelques-uns d'entre eux tout autant que par les pouvoirs publics qui se multipliaient au plus près des marchés de gros. Une géographie de la filière ne peut seulement être envisagée par un traitement géographique de données macro-économiques mais peut donner lieu à une géographie des lieux et des routes, ce qui se passe le long de la chaîne mais aussi ce que l'on ne voit pas à s'arrêtant à cette perspective économique, c'est-à-dire les relations que les sociétés établissent localement à leur espace. 
Nous ne sommes pas allés en Chine ensemble, mais nous sommes partis de ces lieux qui étaient éparpillés dans le monde arabe, au cœur de la Méditerranée ou en Afrique pour savoir d'où provenait ces objets que l'on voyait, et quels étaient les personnes qui faisaient des vas-et-viens entre ces lieux et les alimentaient. L'idée c'était de remonter la filière, et donc d'aller jusqu'à la source, là où ces commerçants achètent leurs marchandises, d'abord dans les marchés de gros des villes ouest-africaines, puis dans le monde arabe et ainsi de suite parfois jusqu'à Dubaï. L'idée c'était de voir quels types de réseaux et de solidarités se mettaient en place entre des lieux et, par exemple, entre des membres d'une famille éclatée entre plusieurs lieux : c'est assez courant maintenant de trouver des familles africaines où il $\mathrm{y}$ a un membre qui habite en Chine, en Europe ou au Canada, mais aussi quelques-uns qui sont entre milieu urbain et milieu rural en Afrique de l'ouest. C'est en essayant de relier tous ces gens et tous ces lieux que l'on voit se dessiner des filières. On peut ainsi passer du microlocal au mondial et voir quels étaient les gens qui impliqués dans ces filières, du petit consommateur final jusqu'au grand producteur, en passant par tous les intermédiaires, qui dans le demi-gros sont souvent des femmes. Et en mettant en lien tout cela, on a pu voir émerger des lieux qui font sens et qui ne sont pas forcément des grands marchés mais peuvent être des petits marchés et/ou des villes secondaires.

Ce travail sur les filières nous a permis aussi de dresser comme une typologie des produits et des objets. Ainsi, il y a deux filières de produits chinois : ceux qui sont produits et achetés en Chine, mais qui sont de piètre qualité, ou du moins considérés comme tels par les consommateurs dans le monde arabe et en Afrique, et puis il y a les produits chinois à destination des marchés européens et qui reviennent sur les marchés africains en produit de seconde main. En Afrique, à prix égal, mieux vaut acheter des produits chinois d'occasion qui ont transité par l'Europe que des neufs venant directement de Chine. C'est là que l'on retrouve les migrants qui remplissent des conteneurs au Havre où à Rotterdam et qui exportent en Afrique et dans le monde arabe. Donc tout vient de Chine, mais sans suivre les mêmes filières et les mêmes routes. Le grand enseignement, c'est qu'alors que l'on pensait que les produits chinois allaient faire disparaitre les marchés de seconde main, ce n'est en fait pas du tout le cas, et cela reste un énorme marché.

À propos des objets

JFS. Lieux, circulations... objets. Ce livre est aussi un livre de géographie des objets (du parpaing de ciment à la mèche de cheveux). L'objet fascine de longue date les anthropologues, les historiens et certains géographes qui en Afrique se sont déjà intéressés à eux. Au-delà de votre étude, quelle doit être la place de l'objet dans la géographie ? Comment l'appréhender?

AC \& OP. Nous utilisons l'objet comme outil pour investiguer. Avec le tournant matériel, on trouvait que c'était intéressant dans ce monde complexe de tenter de le saisir à partir de l'objet. Ce qui nous intéressait, c'était de voir ce que tel ou tel autre objet peut représenter symboliquement et socialement : les objets ont un coût et de ce fait renseignent sur l'économie. Ils sont aussi produits dans un lieu, consommés dans un autre, ils circulent. Les géographes en utilisant les objets peuvent lire de nouveaux rapports sociaux-spatiaux. On devine ainsi des relations plus complexes que celles que l'on peut identifier de prime abord. Des objets peuvent par exemple avoir une forte dimension politique, et la vie politique des objets est questionnée depuis longtemps par les travaux de certains anthropologues. 
Prenons ici le cas du sac de ciment qui est évoqué dans le livre : c'est un objet qui traverse plusieurs frontières et qui, de ce fait, a une dimension politique car on permet de le laisser passer. Ce qui est intéressant, c'est qu'il n'est plus simplement question de production, mais bien de circulation. L'objet circule et le contrôle de la circulation joue un rôle important. La plupart du temps nous avons travaillé sur des réseaux formels, et nous avons pu observer la multiplicité des types et des formes de laisser-passer. Ceux-ci se négocient partout : au niveau supra-étatique par des traités et des accords (comme au sein de la Communauté Économique des États d'Afrique de l'Ouest), mais aussi à des échelles plus fines, aux postes-frontières où tout se renégocie. Il y a là une place pour des pratiques corruptives. Mais il faudrait faire une étude plus approfondie des effets de la numérisation et des réseaux WhatsApp qui limitent de plus en plus ces pratiques, soit les marchandises sont mieux suivies, soit elles ont été payées par différents canaux, numériques. La circulation des objets ne s'accompagne plus nécessairement d'une circulation de monnaie en liquide. Car si personne n'a de compte en banque, tout le monde est bancarisé par les réseaux de téléphonie mobile, à travers lesquels il est possible de payer, et cela participe d'une redéfinition de la place des intermédiaires et des frais.

À propos de l'entrepreneuriat des pauvres

JFS. Si ce livre est une contribution à la géographie de la mondialisation, il pose aussi la question des dynamiques de ses acteurs entrepreneuriaux et des débats que suscite la délicate et très idéologique question de l'entrepreneuriat des pauvres. La conclusion reste relativement prudente sur votre positionnement vis-à-vis de ces nombreux débats idéologiques : comment, au regard de vos travaux envisageriez-vous un positionnement sur la question?

AC \& OP. L'idée centrale du livre, c'est de dire que la mondialisation touche les pauvres, mais certainement pas de dire qu'ils profitent de la mondialisation, bien au contraire. La plupart d'entre eux sont des entrepreneurs par défaut, et sont, tout entrepreneurs qu'ils soient, dans une énorme précarité. En ce sens, nous sommes bien plus proches des théories de Esther Duflo que de Mohamed Yunus ou de Hernando De Soto qui voient des entrepreneurs partout. Le développement passera peut-être par quelques-uns de ces entrepreneurs qui s'en sortent, qui émergent, mais cela ne suffira pas pour développer des sociétés tant le ruissellement est limité. Et il ne faut en effet pas oublier que la très grande majorité de ces entrepreneurs pauvres sont aussi, en même temps, des consommateurs pauvres. En tant que tels, ils ont aussi envie de posséder des choses et cela peut créer beaucoup de frustration et des tensions sociales et politiques. Les équilibres sont précaires tant pour les entrepreneurs / travailleurs que pour les consommateurs, qui sont très bien informés et qui savent ce à quoi ils n'ont pas accès. Avec des contrastes qui sont très marqués et des inégalités qui vont crescendo. Et puis, bien sûr, il ne faut pas oublier que ce sont 4 milliards de consommateurs qui aspirent aussi à être autre chose que des consommateurs.

Nous insistons surtout dans ce livre sur le fait qu'il ne faut pas oublier ces acteurs de la mondialisation, ceux qui la portent modestement, mais aussi et surtout la subissent. 


\section{AUTEURS}

\section{ARMELLE CHOPLIN}

Armelle Choplin, armelle.choplin@unige.ch, est Professeure associée au Département de géographie et environnement, Global Studies Institute de l'Université de Genève. Elle a récemment publié :

- Choplin A., 2019. Cementing Africa: cement flows and city making in the West African corridor (Accra-Lomé-Cotonou-Lagos. Urban Studies. https://doi.org/10.1177/0042098019851949 - Choplin A, Lozivit M., 2019. Mettre un quartier sur la carte : Cartographie participative et innovation numérique à Cotonou (Bénin). Cybergeo: European Journal of Geography [En ligne]. http://journals.openedition.org/cybergeo/32152; DOI : 10.4000/cybergeo

- Choplin A., 2019. Produire la ville en Afrique de l'Ouest : le corridor urbain de Accra à Lagos.

L'Information géographique, vol. 83, p. 85-103. DOI : 10.3917/lig.902.0085.

\section{OLIVIER PLIEZ}

Olivier Pliez, olivier.pliez@cnrs.fr, est directeur de recherche au CNRS et membre de l'UMR ARTDEV. Il a notamment publié :

- Pliez O., 2011. Les cités du désert : des villes sahariennes aux saharatowns. Marseille-Toulouse, IRD-PUM, $164 \mathrm{p}$.

- Bredeloup S., Pliez O. (coord.), 2005. Migrations entre les deux rives du Sahara. Autrepart, $\mathrm{n}^{\circ} 36$, $192 \mathrm{p}$.

- Choplin A., Pliez O., 2015. Inconspicuous Globalization. Articulo. Journal of Urban Research [En ligne], vol. 12. http://journals.openedition.org/articulo/2905

\section{JEAN-FABIEN STECK}

Jean-Fabien Steck, jsteck@parisnanterre.fr, est maître de Conférences HDR à l'Université Paris Nanterr et membre de l'UMR Lavue 7218, Equipe Mosaïques. Il a récemment publié :

- Fourault-Cauët V., Steck J-F., 2018. National Parks in the Urban arena: the interplay of Actors for Incomplete Models of Governance. In Landy F. (ed), From Urban National parks to Natured Cities in the Global South. Springer, Singapore, p. 201-216.

- Dagnono F., Ninot O., Lombard J., Steck J.-F., 2018. Entre la ville et la campagne, entre la brousse et le monde : systèmes de transports et territoires métropolitains en Afrique. In Sanjuan T., Lesourd M., Tallet B. (ed), Tropiques, développement et mondialisation. Pari, L'Harmattan, p. 113-128.

- Steck J.-F., Didier S., Morange M., Rubin M., 2013. Informality, public space and urban governance: An approach through street trading. In Bekker S., Fourchard L. (ed.), Politics and Policies: Governing Cities in Africa. Cape Town, HSRC Press, p. 145 à 167. 